\title{
An Autopsy Case of Acute and Nonalcoholic Thiamine-Deficient Encephalopathy
}

\author{
Yukiko Hata Yui Takeuchi Koshi Kinoshita Naoki Nishida \\ Department of Legal Medicine, Graduate School of Medicine and Pharmaceutical Sciences, University of Toyama, \\ Toyama, Japan
}

\section{Dear Sir,}

Thiamine deficiency can cause a severe neurological disorder known as Wernicke's encephalopathy (WE). While this encephalopathy is considered common in alcoholics [1], only a small number of nonalcoholic cases have been reported [2-5]. The important pathological and radiological features of thiamine-deficient encephalopathy are the selective vulnerability of specific brain regions to pathologic changes [6]. We report a case of autopsy-proven acute nonalcoholic thiamine-deficient encephalopathy without medical treatment antemortem.

\section{Case Report}

A 62-year-old lean man $(163 \mathrm{~cm}, 31.1$ $\mathrm{kg}$, BMI 11.7) was found dead in his room in the supine position. Police examination showed that he had left his employer because of depression 10 years earlier, and had subsequently been withdrawn at home for 7 years before his death. He had no other significant medical history. His family noticed a decrease in food intake and rapid emaciation over the preceding month. However, according to the family, his behavior and mental condition had not changed immediately prior to death. The unwitnessed interval by family was approximately 1 day, and the patient did not have a habit of drinking alcohol.

A medicolegal autopsy revealed severe pyloric stenosis with dilatation of the stomach (fig. 1a, b). Histologic examination showed proliferation and invasion of atypical cells with glandular formation, suggestive of moderately differentiated adenocarcinoma (fig. 1c). Metastasis of tumor cells was seen in many of the circumferential lymph nodes, but not in other organs. Because of the amount of gastric contents filling the upper airway, the direct cause of death was considered to be asphyxia due to aspiration. There was also a small abscess in the right upper lung lobe.

The brain weighed 1,350 g and, macroscopically, no significant atrophy was seen in the cerebrum, cerebellum, or brain stem. On coronal sections of the cerebrum, bilateral mammillary bodies showed petechial and local hemorrhage without obvious brown discoloration and atrophy (fig. 2a). The third ventricle was mildly dilated, and the folia of the superior vermis and dorsomedial portion of the cerebellar hemispheres were not atrophic (fig. 2b).

On light microscopy, fresh petechial hemorrhages were prominent with some foci of proliferation of immature small blood vessels in the mammillary body. Proliferation of vascular endothelial cells with nuclear swelling was also found (fig. 2c), and there was very mild spongiosis without prominent loss of neuronal cells and increasing astrocytes. The number of neurons in the mediodorsal thalamic nuclei and anterior nuclei was well preserved (fig. 2d). In the cerebellum, the Purkinje cells and the molecular layer were unremarkable. Neuronal loss of the Betz cells of the primary motor cortex and the nuclei of the brain stem was not evident, and central chromatolysis of the neuronal cells in pontine and other nuclei was also not evident. Immunohistochemically, there was no inflammation, gliosis, senile plaque, tau, or Lewy pathology.

Biochemical examination of the patient's blood approximately $34 \mathrm{~h}$ after his death revealed that the blood and serum level of vitamin $B_{1}$ was $14.0 \mathrm{ng} / \mathrm{ml}$ (reference range: $20-50 \mathrm{ng} / \mathrm{ml}$ ), vitamin $B_{12}$ was $129 \mathrm{pg} / \mathrm{ml}$ (reference range, 180-914 pg/ $\mathrm{ml}$ ), pyridoxamine was $16.6 \mathrm{ng} / \mathrm{ml}$ (reference range, $<0.6 \mathrm{ng} / \mathrm{ml}$ ), and niacin was $10.5 \mu \mathrm{g} / \mathrm{ml}$ (reference range, 4.7-7.9 $\mu \mathrm{g}$ / $\mathrm{ml}$ ). The blood levels of pyridoxal, pyridoxine, and folic acid were normal, and ethanol was not detected.
Prof Naoki Nishida, MD, $\mathrm{PhD}$ Department of Legal Medicine Graduate School of Medicine and Pharmaceutical Sciences, University of Toyama 2630 Sugitani, Toyama 930-0194 (Japan)

E-Mail nishida@med.u-toyama.ac.jp 


\section{Discussion}

Because it is unclear whether the patients showed the classical triad of WE symptoms (nystagmus, ophthalmoplegia, and severe mentation change), we believe that this case is best described as autopsyproven acute and nonalcoholic 'thiaminedeficient encephalopathy'. It is known that chronic excessive alcohol intake does not result in WE if there is adequate thiamine intake [7]. Many conditions, such as prolonged intravenous feeding, malignancy, anorexia nervosa, thyrotoxicosis, enteritis, malabsorption, hemodialysis, uremia, HIV infection, and gastroplasty, are reported causes of nonalcoholic WE $[1,4]$. Among the possible gastrointestinal factors, gastrojejunostomy is considered to be a common cause, and untreated advanced gastric cancer may be an additional rare cause [3]. Pyloric obstruction may be a major cause of thiamine deficiency in our case.

Macroscopic and microscopic features are considered to be dependent on the stage and the severity of WE [8]. Pathologically, the phases of WE are classified as acute, chronic, or acute on chronic. Chronic lesions exhibit swelling of astrocytes, minimal loss of neurons, a decrease in myelinated fibers, activated microglia, and reactive astrogliosis $[1,5]$. Our case did not show any of these pathological findings, but did show apparent extravasation of red blood cells into the perivascular space, swelling of endothelial cells, and mild proliferation of immature vessels as acute lesions. Such pathological features may suggest a rapid progression of thiamine deficiency, but this patient did not show any apparent neurological symptoms at least 1 day before death.

The other pathological feature of this case is that the lesions were localized to both mammillary bodies. This may be a vulnerable region in thiamine deficiency, as the mammillary bodies are reportedly the most frequently affected structure $[8$, 9]. Periventricular areas, such as the thalamus, hypothalamus, and the periaqueductal gray matter at the level of the 3rd cranial nerve and reticular formation of the brain stem, also have a reportedly high incidence $[1,2,5,8,9]$. Harper [9] reported that $17 \%$ of 131 patients were pathologically classified as the acute phase, as in our case; however, the anatomical distribution of the lesions in the study was not clearly noted. Our case may suggest that the lesions in nonalcoholic WE progress in
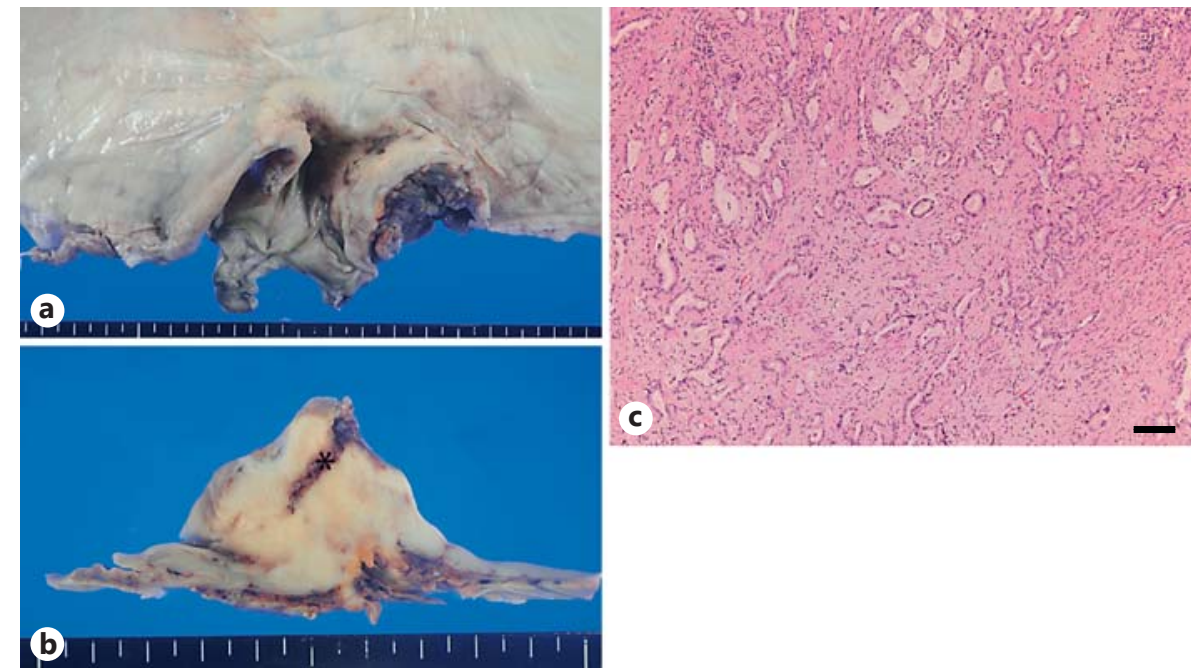

C

Fig. 1. Pathological findings in the stomach: severe pyloric stenosis (a), vertical section of the pyloric ring showing severe narrowing of the inner cavity (asterisk) (b), and microscopic appearance of the stomach showing adenocarcinoma proliferation (scale bar: 100 $\mu \mathrm{m})(\mathbf{c})$.
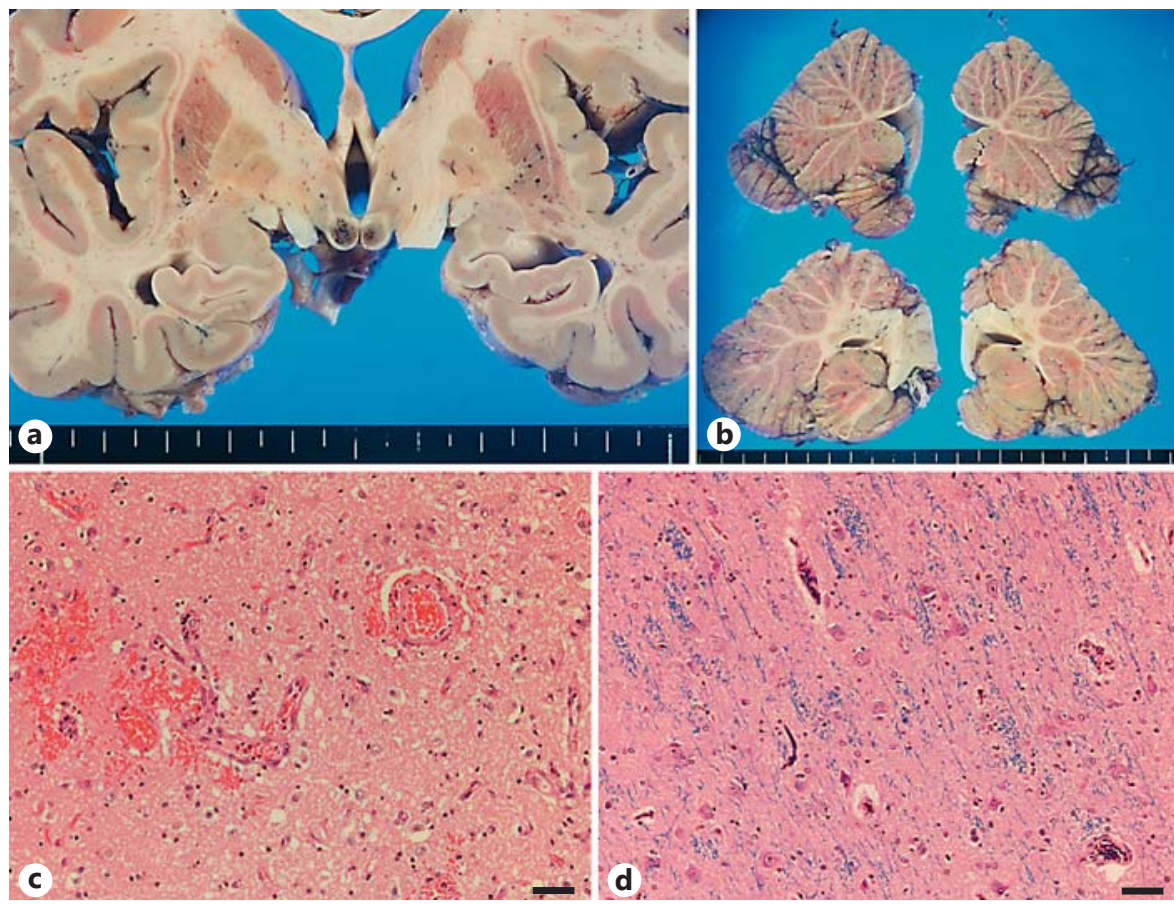

Fig. 2. Macro- and microscopic appearance of the brain: petechial hemorrhage is seen in both mammillary bodies and obvious discoloration and atrophy are not seen (a), atrophy of the cerebellar vermis is not evident (b), high-power view of the mammillary bodies showing fresh hemorrhage with proliferation of small vessels (c), and high-power view of the medial dorsal nucleus of the thalamus showing no obvious neuronal loss (d). c, d Scale bars: $50 \mu \mathrm{m}$. 
stages; further pathological examination of acute cases or comparisons between alcoholic and nonalcoholic cases may be important. In addition, our case did not show any pathological change in the cerebellum. There may be only a minimal relationship between thiamine deficiency and the occurrence of cerebellar degeneration.

\section{References}

1 Sechi G, Serra A: Wernicke's encephalopathy: new clinical settings and recent advances in diagnosis and management. Lancet Neurol 2007;6:442-455.

-2 Gui QP, Zhao WQ, Wang LN: Wernicke's encephalopathy in nonalcoholic patients: clinical and pathologic features of three cases and literature reviewed. Neuropathology 2006;26: 231-235.

3 Weidauer S, Rösler A, Zanella FE, et al: Diffusion-weighted imaging in Wernicke encephalopathy associated with stomach cancer: case report and review of the literature. Eur Neurol 2004;51:55-57.
In clinical practice, the diagnosis of thiamine-deficient encephalopathy is considered difficult, especially when patients are in a comatose state and nonalcoholic [3, 10]. It may be important for pathologists to consider thiamine-deficient encephalopathy as a cause of sudden unexpected death, even if a case had no history of alcohol misuse or the typical clinical findings of WE.

\section{Disclosure Statement}

The authors declare that they have no conflicts of interest.
4 Tanaka K, Aoki M, Hamada Y, et al: Wernicke's encephalopathy caused by pyloric stenosis after endoscopic submucosal dissection. Gastrointest Endosc 2009;69:1170-1171.

$\checkmark 5$ Caso F, Fiorino A, Felautano M, et al: Treatment of Wernicke's encephalopathy with high dose of thiamine in a patient with pyloric sub-stenosis: description of a case. Neurol Sci 2010;31:859-861.

6 Jhala SS, Hazell AS: Modeling neurodegenerative disease pathophysiology in thiamine deficiency: consequences of impaired oxidative metabolism. Neurochem Int 2011;58:248-260.

7 Zimitat C, Kril J, Harper CG, et al: Progression of neurological disease in thiamin-deficient rats is enhanced by ethanol. Alcohol 1990;7:493-501.
8 Victor M, Adams RD, Collins GH: The Wernicke-Korsakoff syndrome. A clinical and pathological study of 245 patients, 82 with postmortem examinations. Contemp Neurol Ser 1971;7:1-206.

9 Harper C: The incidence of Wernicke's encephalopathy in Australia - a neuropathological study of 131 cases. J Neurol Neurosurg Psychiatry 1983;46:593-598.

10 Suzuki S, Ichijo M, Fujii H, et al: Acute Wernicke's encephalopathy: comparison of magnetic resonance images and autopsy findings. Intern Med 1996;35:831-834. 